\title{
The need for efficient long-term neuroleptic treatment in schizophrenic patients and the place of long acting injectable antipsychotics
}

\author{
Hans-Jurgen Möller
}

\author{
From $1^{\text {st }}$ International Congress on Neurobiology and Clinical Psychopharmacology and European \\ Psychiatric Association Conference on Treatment Guidance \\ Thessaloniki, Greece. 19-22 November 2009
}

Schizophrenia is a chronic disorder with a high risk of poor outcome in terms of symptoms and social functioning and possibly also progressive brain alterations. The relapse rate is high and each relapse can induce further aggravations. Thus, long-term treatment with the highest degree of effectiveness should be provided to the patients. Amongst others, the suitable drug for the individual patient has to be selected as well as the high risk of non-compliance to be carefully considered.

All the available evidence from randomised controlled studies indicates that antipsychotic medications substantially reduce the risk of relapse. The lowest dose should be chosen at which preferably no side effects occur, the risk of relapse seems to be optimally reduced and, if symptoms are still present, suppression of these is optimised. Side effects have to be assessed and, if necessary, pharmacotherapy has to be adjusted.

Despite several methodological design issues, secondgeneration antipsychotics have proven superior efficacy in preventing relapse to FGAs. Available studies of the specific agents supply evidence for periods of up to 2 years. Due to the decreased risk of EPS, especially tardive dyskinesia and the superior efficacy in improving negative, cognitive and depressive symptoms, secondgeneration antipsychotics should be preferred in longterm treatment.

Given all the known problems in compliance and discontinuation, which were underlined in recent years by the CATIE and the EUFEST study, depot preparations should be considered for optimum effectiveness in preventing relapse. Altogether, randomised, control-group studies to determine the long-term advantages of depot preparations of atypical neuroleptics compared to depots of typical neuroleptics are still lacking. However, the huge database for long acting injectable risperidone is so convincing in terms of efficacy, tolerability and effectiveness that its special place in the long-term treatment of schizophrenia becomes obvious.

The target strategy in long-term treatment of schizophrenia should be a combination of long-term antipsychotic treatment and psycho- and sociotherapeutic procedures, so that the relapse rate is further reduced and the course of disease can be further improved.

Published: 22 April 2010

\section{References}

1. Möller HJ: Course and long-term treatment of schizophrenic psychoses. Pharmacopsychiatry 2004, 37(Suppl 2):126-35.

2. Möller HJ: Long-acting risperidone: focus on safety. Clin Ther 2006, 28(5):633-51.

3. Möller HJ: Long-acting injectable risperidone for the treatment of schizophrenia: clinical perspectives. Drugs 2007, 67(11):1541-66.

4. Möller HJ: Systematic of psychiatric disorders between categorical and dimensional approaches: Kraepelin's dichotomy and beyond. Eur Arch Psychiatry Clin Neurosci 2008, 258(Suppl 2):48-73.

doi:10.1186/1744-859X-9-S1-S68

Cite this article as: Möller: The need for efficient long-term neuroleptic treatment in schizophrenic patients and the place of long acting injectable antipsychotics. Annals of General Psychiatry 2010 9(Suppl 1):S68. 